Arakelian: Seyfert galaxies do not show a concentration towards the Coma cluster but the statistics are very small.

\title{
REMARKS ON THE ANGULAR DISTRIBUTION OF MARKARIAN GALAXIES
}

\section{Piotr Flin and Marek Urbanik}

Lists I-IX of Markarian galaxies have been used as the basic observational material. An attempt has been made to form a statistically complete sample. Two regions (region I $6.8<\alpha<14.2,41^{\circ}<\delta<77^{\circ}$, region II $6.8<\alpha<13$ h 8 , $5^{\circ}<\delta<32^{\circ}$ ) which appeared to be roughly complete have been carefully examined. After preliminary tests the first two hours in R.A. of the region II were removed from the analysis. The method of statistical reduction (F1in et al. 1974, A. Zieba 1975) and the maximum likelihood estimate of the number-magnitude counts (Crawford et a1. 1970) were applied as a combined test in their practical version (Urbanik 1976,1977). We have examined the whole region, as well as a number of smaller sub-regions varying in size and position. The angular sizes investigated ranged from $1^{\circ}$ up to $30^{\circ}$. Our analysis of 15 galaxy sets led to the following conclusions.

A much higher tendency toward clustering in region II in comparison with region I is accompanied by a significant decrease of surface density and flattening of number-magnitude counts. This effect varies across region II. This situation remains unchanged after restricting the analysis to galaxies brighter than $16^{\mathrm{m}}$. It has been found that multiple re-surveying of some small fields causes a sharp increase of density within them. These facts resemble the behaviour of radio source counts when investigations are carried out below the completeness limit of the radio survey. Therefore we infer that the completeness of the region II is considerably worse than that of the region I. Moreover the incompleteness is independent of the strength of the UV. The properties of the distribution of galaxies in region I do not differ qualitatively from those obtained for fainter normal galaxies (S. Zieba 1977). This similarity disappears in region II. In the light of these conclusions it seems that any large-scale statistical analysis of Markarian galaxies requires improvement in the completeness of the observational data.

\section{REFERENCES}

Crawford, D.F., Jauncey, D.L. and Murdoch, M.S., 1970. Astroph. J.,

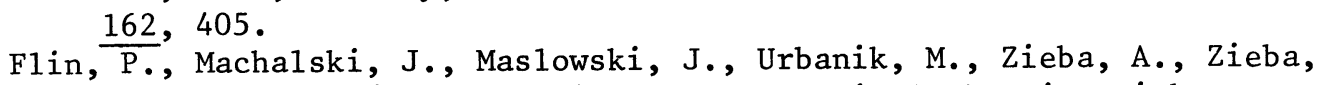
S., 1974. In "Confrontation of Cosmological Theories with Observational Data" (ed. M. S. Longair), p.121 (D. Reide1, Dordrecht).

Urbanik, M., 1976. Acta Cosmologica, 5, 97. Urbanik, M., 1977. Thesis, Jagiellonian University. Zieba, A., 1975. Acta Cosmologica, 3, 75. Zieba, S., 1977. Rozprawy habilitacyjne U.J., No.2, Krakow. 


\section{DISCUSSION}

Huchra: Can you estimate a magnitude limit for the completeness of the Markarian Surveys?

Urbanik: We estimate the limiting magnitude for completeness in region II to be about $14^{\mathrm{m}}$ or even worse, and about $15^{\mathrm{m}}$ in region $\mathrm{I}$.

Heidmann: Cannot these limiting magnitudes for completeness be simply obtained from the $\log$ (number) versus magnitude counts?

Urbanik: The limiting magnitude for completeness can, of course, be obtained from such counts. We did not make counts, because we were not specially interested in a knowledge of limiting magnitude for each region individually. We were interested in the mutual relations between changes of the tendency toward clustering, average surface density and the slope of the mean number-magnitude counts.

\section{SUPERCLUSTERING OF GALAXIES}

M. Kalinkov, V. Dermendjiev, B. Staikov

I. Kaneva, B. Tomov, and K. Stavrev

A new type of catalogue of extragalactic objects is nearing completion in our department. The catalogue is on magnetic tape and is a compilation of data for galaxies and cluster of galaxies, together with references, and for counts of galaxies. At present this Metacatalogue contains about $3 \times 10^{5}$ entries - Abe11 and Zwicky clusters, Zwicky galaxies, Lick counts, Jagellonian counts and some others. The first two files of the catalogue can be obtained on request from the Centre de Données Stellaire at Strasbourg.

The Metacatalogue enables us to investigate the large scale distribution of galaxies. A process for searching for second-order clusters of galaxies has been developed. The process consists of smoothing and filtering two-dimensional discrete fields, defined on a lattice, the trend being attenuated beforehand. The process is described by Kalinkov, M. (1974, Proc. First Europ. Astron. Meet., 3, 142; 1975, Mem. Soc. Astron. Ital., 45, 639; 1977, Highlights of Astronomy, 4, part I, 279), by Kalinkov, M., Stavrev, K., Kaneva, I., Dermendjiev, V. (1976, in Stars and Galaxies from Observational Point of View, Tbilisi, 309) and by Kalinkov, M., Kaneva, I., Stavrev, K., Tomov, B., Vlahova, K., and Yanev, K. (1976, C. R. Acad. Bulg. Sci., 29, 453).

Some examples of the latest (1977) results are given in Figures $1-3$.

Figure 1 shows a medium scale filtering for all Zwicky clusters (northern galactic hemisphere). The North Galactic Pole is at the centre and the point of view is $\ell=315^{\circ}$. At least $60 \%$ of al1 condensations are real second-order clusters with characteristic sizes of 